\title{
ISOTROPIC INFINITELY DIVISIBLE PROCESSES ON COMPACT SYMMETRIC SPACES
}

\author{
BY RAMESH GANGOLLI
}

Communicated by Edwin Hewitt, January 25, 1963

Let $G$ be a connected compact Lie group, $K$ a compact subgroup, such that $G / K$ is a Riemannian symmetric homogeneous space. (See [1] for terminology and notation.) We fix once and for all a $G$-invariant metric on $G / K . D(G / K)$ will stand for the algebra of those differential operators on $C^{\infty}(G / K)$ which are invariant under the action of $G$. $\mathbf{S}(K \backslash G / K)$ stands for the semi-group, under convolution as product, of probability measures on $G$ which are bi-invariant under the action of $K$. It is known [2] that $S$ is a commutative semi-group. Let $\phi_{n}(x), n=0,1,2, \cdots$ be the $K$-spherical functions on $G[1$, pp. $398 \mathrm{ff}$.]. For $\mu \in S$, we define the Fourier coefficients $\hat{\mu}(n)$ $=\int_{G} \phi_{n}(x) d \mu(x)$. A measure $\mu \in S$ is said to be infinitely divisible if for each positive integer $k, \mu=\nu^{k}$ for some $\nu \in S$. The purpose of this note is to set down characterizations of such measures. Our main theorem is

THEOREM 1. Let $\mu \in S$ be such that $\hat{\mu}(n) \neq 0$ for any $n$. Then $\mu$ is infinitely divisible if and only if

$$
\hat{\mu}(n)=\exp -\left(\lambda_{n}+\int_{G-\{e\}}\left(1-\phi_{n}(x)\right) M(d x)\right)
$$

where $M(d x)$ is a non-negative measure on $G$, bi-invariant under $K$ and such that $\int_{G} r^{2} M(d x)<\infty, r$ being the distance of $x \in G$ from $e$; and $\lambda_{n}$ is the eigenvalue corresponding to the eigenfunction $\phi_{n}$ of an elliptic, second order operator $D$ in $\mathbf{S}(G / K)$. Further, $D$ and $M$ are uniquely determined by $\mu$.

Let $\mu \in S$ be such that $\hat{\mu}(n) \neq 0$ for any $n$. Call $\mu$ a generalized limit for each $n, \hat{\mu}(n)=\lim _{j \rightarrow \infty} \prod_{r=1}^{k_{j}} \hat{\mu}_{j r}(n)$ with $\mu_{j r} \in S,\left|\hat{\mu}_{j r}(n)-1\right| \rightarrow 0$ as $j \rightarrow \infty$ uniformly for $1 \leqq r \leqq k_{j}$.

THEOREM 2. The class of infinitely divisible measures of Theorem 1 coincides with the class of generalized limits.

As a corollary to Theorem 1, we can state results about the Fourier coefficients $\hat{\mu}_{t}(n)$ of one-parameter subsemigroups of $S(K \backslash G / K)$ or what is the same thing, describe $K$-isotropic stochastic processes on 
$G / K$ which are homogeneous in space and have stationary independent increments.

We might remark that Theorem 1 is a special case of a theorem of Hunt [3, Theorem 5.1]. However, our methods are more direct than those of Hunt, due to the special nature of our set-up and thus do not require the formidable machinery of that paper.

We have obtained descriptions also of those infinitely divisible measures $\mu \in S$ which do not satisfy the condition $\hat{\mu}(n) \neq 0$ of Theorem 1. The proofs of these will be published elsewhere along with probabilistic descriptions of the path functions of the stochastic process associated with measures such as those of Theorem 1.

As a result of specializing our results to two-point homogeneous spaces [1] we can re-derive results of Bochner in [4], where $G=S O(n+1), K=S O(n)$ and the $\phi_{k}$ correspond to the ultraspherical polynomials. An interesting and hitherto unobserved specialization of our results is when $G=\mathbf{S} U(n+1), K=\boldsymbol{U}(n)$. Then $G / K$ is a complex projective space. The spherical functions $\phi_{k}$ turn out to be Jacobi polynomials $P_{k}^{((n-1) / 2,0)}$ in Szegö's [5] notation, and we are able to state results for these similar to Bochner's results for the ultraspherical polynomials mentioned above.

Remark added in proof, March 15, 1963. The assumption that for each $x \in G, x=k x^{-1} k^{\prime}$ for some $k, k^{\prime} \in K$, is necessary for the truth of Theorem 1 as stated, but was inadvertently omitted. However, since the time of writing the above, we have succeeded in relaxing this assumption as also the assumption that $G$ is compact, and replaced the latter by the assumption that $G$ be a connected semi-simple Lie group. The representation formula of Theorem 1 has, of course, to appear in a modified form.

\section{BIBLIOGRAPHY}

1. S. Helgason, Differential geometry and symmetric spaces, Academic Press, New York, 1962.

2. R. Godement, Introduction a travaux de A. Selberg, Exposé 144, Seminaire Bourbaki, Paris, 1957.

3. G. A. Hunt, Semi-groups of measures on Lie groups, Trans. Amer. Math. Soc. 81 (1956), 264-293.

4. S. Bochner, Sturm-Liouville and heat equations whose eigenfunctions are ultraspherical polynomials or associated Bessel functions, Proceedings of the Conference on Differential Equations, University of Maryland, 1956.

5. G. Szegö, Orthogonal polynomials, Amer. Math. Soc. Colloq. Publ. Vol. 24, rev. ed., Amer. Math. Soc., Providence, R. I., 1959.

UNIVERSITY OF WASHINGTON 